\title{
Two-Step Hybrid Block Method for Solving Nonlinear Jerk Equations
}

\author{
Bothayna S. H. Kashkari' ${ }^{1}$, Sadeem Alqarni ${ }^{2,3}$ \\ ${ }^{1}$ Department of Mathematics, Faculty of Science, University of Jeddah, Jeddah, KSA \\ ${ }^{2}$ Department of Mathematics, Faculty of Science, King Abdulaziz University, Jeddah, KSA \\ ${ }^{3}$ Department of Mathematics, Faculty of Science, Al-Baha University, Al-Baha, KSA \\ Email: bskashkari@uj.edu.sa, saalqarni@bu.edu.sa
}

How to cite this paper: Kashkari, B.S.H. and Alqarni, S. (2019) Two-Step Hybrid Block Method for Solving Nonlinear Jerk Equations. Journal of Applied Mathematics and Physics, 7, 1893-1910.

https://doi.org/10.4236/jamp.2019.78130

Received: July 24, 2019

Accepted: August 23, 2019

Published: August 26, 2019

Copyright (c) 2019 by author(s) and Scientific Research Publishing Inc. This work is licensed under the Creative Commons Attribution International License (CC BY 4.0).

http://creativecommons.org/licenses/by/4.0/

\begin{abstract}
In this paper, a block method with one hybrid point for solving Jerk equations is presented. The hybrid point is chosen to optimize the local truncation errors of the main formulas for the solution and the derivative at the end of the block. Analysis of the method is discussed, and some numerical examples show that the proposed method is efficient and accurate.
\end{abstract}

\section{Keywords}

Nonlinear Jerk Equations, Hybrid Block Method, Zero Stable, Consistent, Convergent

\section{Introduction}

Jerk is the rate of acceleration change in physics; that is, the time derivative of acceleration, and as such the second velocity derivative, or the third time position derivative. The jerk is important in several mechanics and acoustics applications. The Jerk vector is here resolved into tangential-normal and radial-transverse components for planar motion, and the normal component is expressed as an affine differential invariant recognized as the aberrancy. Several geometric properties of the Jerk vector are established for plane motion using known aberrancy properties of curves [1].

Nonlinear third-order differential equations, known as nonlinear Jerk equations, involving the third temporal displacement derivative, are of great interest in analyzing some structures which exhibit rotating and translating movements, such as robots or machine tools, where excessive Jerk leads to accelerated wear of transmissions and bearing elements, noisy operations and large contouring errors in discontinuities (such as corners) in the machining path [2]. 
Many authors have studied the numerical solutions of the Jerk equation, harmonic balance approach to periodic solutions is used in [3], in [4] they have written the high-order ordinary differential equation in terms of its differential invariants. New algorithm for the numerical solutions of nonlinear third-order differential equations was used jacobi-gauss collocation method in [5], He's variational iteration method was used in [6] for nonlinear Jerk equations. Modified harmonic balance method was used for nonlinear Jerk equations in [7]. In this paper, we consider a Jerk equation of the form

$$
y^{\prime \prime \prime}=J\left(y, y^{\prime}, y^{\prime \prime}\right) \text {. }
$$

With initial conditions $y(0)=0, y^{\prime}(0)=B, y^{\prime \prime}(0)=0$. The most general function of Jerk, which is invariant in time-reversal and space-reversal and only nonlinear cubic, can be written as

$$
y^{\prime \prime \prime}+\alpha y y^{\prime} y^{\prime \prime}+\beta y^{\prime} y^{\prime \prime 2}+\delta y^{2} y^{\prime}+\epsilon y^{\prime 3}+\gamma y^{\prime}=0,
$$

where the parameters are $\alpha, \beta, \delta, \epsilon$ and $\gamma$ is constants. The current work is motivated by optimizing local truncation errors in order to find a hybrid point in a two-step block method to have the most accurate solution for Jerk Equation (1). We organize this paper as follows: The next section illustrates the method derivation, Section 3 presents the analysis of the method involving order four, Section 4 presents the numerical examples showing the productivity of the new technique when it is contrasted with the different strategies proposed in the scientific writing.

\section{Methodology}

To derive two steps hybrid block method with one off-step point we have a polynomial of degree 6 as follows:

$$
y(x) \simeq p(x)=\sum_{j=0}^{6} a_{j} x^{j},
$$

with third derivative given by

$$
y^{\prime \prime \prime}(x) \simeq p^{\prime \prime \prime}(x)=\sum_{j=3}^{6} j(j-1)(j-2) a_{j} x^{j-3} .
$$

Substituting Equation (4) into Equation (3) gives

$$
f\left(x, y, y^{\prime}, y^{\prime \prime}\right)=\sum_{j=3}^{6} j(j-1)(j-2) a_{j} x^{j-3} .
$$

By interpolating Equation (3) at $x_{m+j}, j=0, r, 1$ and collocating Equation (5) at $x_{m+j}, j=0, r, 1,2$ we get a system of equations written in the matrix form

$$
\left[\begin{array}{ccccccc}
1 & x_{m} & x_{m}^{2} & x_{m}^{3} & x_{m}^{4} & x_{m}^{5} & x_{m}^{6} \\
1 & x_{m+r} & x_{m+r}^{2} & x_{m+r}^{3} & x_{m+r}^{4} & x_{m+r}^{5} & x_{m+r}^{6} \\
1 & x_{m+1} & x_{m+1}^{2} & x_{m+1}^{3} & x_{m+1}^{4} & x_{m+1}^{5} & x_{m+1}^{6} \\
0 & 0 & 0 & 6 & 24 x_{m} & 60 x_{m}^{2} & 120 x_{m}^{3} \\
0 & 0 & 0 & 6 & 24 x_{m+r} & 60 x_{m+r}^{2} & 120 x_{m+r}^{3} \\
0 & 0 & 0 & 6 & 24 x_{m+1} & 60 x_{m+1}^{2} & 120 x_{m+1}^{3} \\
0 & 0 & 0 & 6 & 24 x_{m+2} & 60 x_{m+2}^{2} & 120 x_{m+2}^{3}
\end{array}\right]\left[\begin{array}{c}
a_{0} \\
a_{1} \\
a_{2} \\
a_{3} \\
a_{4} \\
a_{5} \\
a_{6}
\end{array}\right]=\left[\begin{array}{c}
y_{m} \\
y_{m+r} \\
y_{m+1} \\
f_{m} \\
f_{m+r} \\
f_{m+1} \\
f_{m+2}
\end{array}\right] \text {, }
$$


Solving the above system (by using Gaussian elimination method for solving the system of linear equations [8]) gives us the coefficients of the polynomial $a_{j}, j=0,1, \cdots, 6$.

By making the substitution $x=x_{m}+t h$, the polynomial in Equation (3) may be written in the form:

$$
p\left(x_{m}+t h\right)=\alpha_{0} y_{m}+\alpha_{r} y_{m+r}+\alpha_{1} y_{m+1}+h^{3}\left(\beta_{0} f_{m}+\beta_{r} f_{m+r}+\beta_{1} f_{m+1}+\beta_{2} f_{m+2}\right)(6)
$$

where

$$
\begin{gathered}
\alpha_{0}=-\frac{(r-t)(t-1)}{r}, \\
\alpha_{r}=\frac{t(t-1)}{r(r-1)}, \\
\alpha_{1}=\frac{t(r-t)}{r-1}, \\
\beta_{0}=-\frac{h^{3}}{240 r}\left(t ( t - 1 ) \left(r^{4}-8 r^{3}+22 r^{2}-2 r t^{3}+13 r t^{2}\right.\right. \\
\beta_{r}=-\frac{\left.\left.27 r t-5 r+t^{4}-5 t^{3}+5 t^{2}+5 t\right)\right),}{120 r\left(r^{2}-3 r+2\right)}\left(t(t-1)\left(r^{4}-5 r^{3}+5 r^{2}+5 r-t^{4}+5 t^{3}-5 t^{2}-5 t\right)\right), \\
\beta_{1}=-\frac{h^{3}}{120(r-1)}\left(t ( t - 1 ) \left(-r^{4}+5 r^{3}+5 r^{2}+2 r t^{3}-8 r t^{2}\right.\right. \\
\left.\left.-8 r t-3 r-t^{4}+3 t^{3}+3 t^{2}+3 t\right)\right), \\
\beta_{2}=\frac{h^{3}}{240(r-2)}\left(t ( t - 1 ) \left(-r^{4}+2 r^{3}+2 r^{2}+2 r t^{3}-3 r t^{2}\right.\right. \\
\left.\left.-3 r t-r-t^{4}+t^{3}+t^{2}+t\right)\right) .
\end{gathered}
$$

Now, by evaluating the solution approximation at the point $x_{m+2}$ :

$$
\begin{aligned}
y_{m+2}= & -\frac{r-2}{r} y_{m}+\frac{2}{r(r-1)} y_{m+r}+\frac{2 r-4}{r-1} y_{m+1} \\
& -\frac{h^{3}\left(r^{4}-8 r^{3}+22 r^{2}-23 r+6\right)}{120 r} f_{m}+\frac{h^{3}\left(-r^{2}+2 r+3\right)}{60 r} f_{m+r} \\
& -\frac{h^{3}\left(-r^{3}+4 r^{2}+9 r-26\right)}{60} f_{m+1}+\frac{h^{3}\left(-r^{3}+2 r+1\right)}{120} f_{m+2} .
\end{aligned}
$$

Assess the approximation at the point of first derivative $x_{m+2}$ :

$$
\begin{aligned}
h y_{m+2}^{\prime}= & -\frac{r-3}{r} y_{m}+\frac{3}{r(r-1)} y_{m+r}+\frac{r-4}{r-1} y_{m+1} \\
& -\frac{h^{3}\left(3 r^{4}-24 r^{3}+66 r^{2}-67 r+12\right)}{240 r} f_{m} \\
& -\frac{h^{3}\left(r^{4}-5 r^{3}+5 r^{2}+5 r-4\right)}{\left(40 r\left(r^{2}-3 r+2\right)\right)} f_{m+r}
\end{aligned}
$$




$$
\begin{aligned}
& -\frac{h^{3}\left(-3 r^{4}+15 r^{3}+15 r^{2}-137 r+116\right)}{120(r-1)} f_{m+1} \\
& +\frac{h^{3}\left(-r^{4}+2 r^{3}+2 r^{2}+3 r-12\right)}{80(r-2)} f_{m+2} .
\end{aligned}
$$

Evaluate the second derivative approximation at the point $x_{m+2}$ :

$$
\begin{aligned}
h^{2} y_{m+2}^{\prime \prime}= & \frac{2}{r} y_{m}+\frac{2}{r(r-1)} y_{m+r}-\frac{2}{r-1} y_{m+1} \\
& +\frac{h^{3}\left(-r^{4}+8 r^{3}-22 r^{2}+18 r+5\right)}{120 r} f_{m} \\
& -\frac{h^{3}\left(r^{4}-5 r^{3}+5 r^{2}+5 r+5\right)}{\left(60 r\left(r^{2}-3 r+2\right)\right)} f_{m+r} \\
& -\frac{h^{3}\left(-r^{4}+5 r^{3}+5 r^{2}-75 r+77\right)}{60(r-1)} f_{m+1} \\
& +\frac{h^{3}\left(-r^{4}+2 r^{3}+2 r^{2}+42 r-81\right)}{120(r-2)} f_{m+2} .
\end{aligned}
$$

We choose to optimize the local truncation errors in the Equation (8), Equation (9) and Equation (10) to determine appropriate values for $r$ Equation (8), Equation (9) and Equation (10). This choice at the end of the block $y_{m+2}, y_{m+2}^{\prime}$ and $y_{m+2}^{\prime \prime}$, which result respectively in

$$
\begin{aligned}
& \mathcal{L}\left(y\left(x_{m+2} ; h\right)\right) \\
= & -\frac{h^{7} y^{(7)}\left(x_{m}\right)}{10080}\left(3 r^{5}-18 r^{4}+24 r^{3}+24 r^{2}-53 r+10\right) \\
& \quad-\frac{h^{8} y^{(8)}\left(x_{m}\right)}{100800}\left(9 r^{6}-33 r^{5}-47 r^{4}+205 r^{3}+51 r^{2}-327 r+42\right), \\
\mathcal{L}\left(y^{\prime}\left(x_{m+2} ; h\right)\right) & \frac{h^{7} y^{(7)}\left(x_{m}\right)}{20160}\left(-9 r^{5}+54 r^{4}-72 r^{3}-72 r^{2}+117 r+44\right) \\
+ & \frac{h^{8} y^{(8)}\left(x_{m}\right)}{201600}\left(-27 r^{6}+99 r^{5}+141 r^{4}-615 r^{3}-237 r^{2}+729 r+556\right), \\
& \mathcal{L}\left(y^{\prime \prime}\left(x_{m+2} ; h\right)\right) \\
= & -\frac{h^{7} y^{(7)}\left(x_{m}\right)}{3360}\left(r^{5}-6 r^{4}+8 r^{3}+8 r^{2}+8 r-41\right) \\
& -\frac{h^{8} y^{(8)}\left(x_{m}\right)}{100800}\left(9 r^{6}-33 r^{5}-47 r^{4}+205 r^{3}+205 r^{2}+135 r-1195\right) .
\end{aligned}
$$

Determine the $r$ values equating to zero the $h^{7}$ coefficients in the local truncation error formulas in Equation (11), and we obtain the system

$$
\begin{aligned}
& -3 r^{5}+18 r^{4}-24 r^{3}-24 r^{2}+53 r-10=0, \\
& -9 r^{5}+54 r^{4}-72 r^{3}-72 r^{2}+117 r+44=0,
\end{aligned}
$$




$$
-r^{5}+6 r^{4}-8 r^{3}-8 r^{2}-8 r+41=0 .
$$

By solving Equation (12) and substituting $r^{2}$ s in Equation (11) we can choose the value $r$ that gives the least truncation errors

$$
r=\frac{161757+\sqrt{36610681841}}{199636} .
$$

Substituting $r$ in the local truncation errors gives:

$$
\begin{gathered}
\mathcal{L}\left(y\left(x_{m} ; h\right)\right) \approx \frac{2 h^{7} y^{(7)}}{211915}+O\left(h^{8}\right) \\
\mathcal{L}\left(y^{\prime}\left(x_{m} ; h\right)\right) \approx-\frac{17 h^{8} y^{(8)}}{107192}+O\left(h^{9}\right) \\
\mathcal{L}\left(y^{\prime \prime}\left(x_{m} ; h\right)\right) \approx \frac{-26 h^{7} y^{(7)}}{84685}+O\left(h^{8}\right)
\end{gathered}
$$

To obtain a two-step hybrid block solution method for solving Equation (1), we evaluate $p^{\prime}(x), p^{\prime \prime}(x)$ at the points $x_{m}, x_{m+r}, x_{m+1}$, we get the following block of six equations

$$
\begin{aligned}
& h y_{m}^{\prime}=-\frac{r+1}{r} y_{m}-\frac{1}{r(r-1)} y_{m+r}+\frac{r}{r-1} y_{m+1} \\
& +\frac{h^{3}\left(r^{3}-8 r^{2}+22 r-5\right)}{240} f_{m} \\
& +\frac{h^{3}\left(r^{3}-5 r^{2}+5 r+5\right)}{120\left(r^{2}-3 r+2\right)} f_{m+r} \\
& +\frac{h^{3} r\left(-r^{3}+5 r^{2}+5 r-3\right)}{120(r-1)} f_{m+1} \\
& -\frac{h^{3} r\left(-r^{3}+2 r^{2}+2 r-1\right)}{240(r-2)} f_{m+2}, \\
& h^{2} y_{m}^{\prime \prime}=\frac{2}{r} y_{m}+\frac{2}{r(r-1)} y_{m+r}-\frac{2}{r-1} y_{m+1} \\
& -\frac{h^{3}\left(r^{4}-8 r^{3}+22 r^{2}-28 r+10\right)}{120 r} f_{m} \\
& -\frac{h^{3}\left(r^{4}-5 r^{3}+5 r^{2}+5 r-10\right)}{60 r\left(r^{2}-3 r+2\right)} f_{m+r} \\
& -\frac{h^{3}\left(-r^{4}+5 r^{3}+5 r^{2}-35 r+22\right)}{60(r-1)} f_{m+1} \\
& +\frac{h^{3}\left(-r^{4}+2 r^{3}+2 r^{2}-8 r+4\right)}{120(r-2)} f_{m+2}, \\
& h y_{m+r}^{\prime}=\frac{r-1}{r} y_{m}+\frac{2 r-1}{r(r-1)} y_{m+r}-\frac{r}{r-1} y_{m+1} \\
& +\frac{h^{3}\left(2 r^{4}-13 r^{3}+28 r^{2}-22 r+5\right)}{240} f_{m}
\end{aligned}
$$




$$
\begin{aligned}
& +\frac{h^{3}\left(4 r^{3}-15 r^{2}+10 r+5\right)}{120(r-2)} f_{m+r} \\
& +\frac{h^{3} r\left(-2 r^{3}+7 r^{2}+2 r-3\right)}{120} f_{m+1} \\
& +\frac{h^{3} r\left(2 r^{4}-5 r^{3}+2 r^{2}+2 r-1\right)}{240(r-2)} f_{m+2}, \\
& h^{2} y_{m+r}^{\prime \prime}=\frac{2}{r} y_{m}+\frac{2}{r(r-1)} y_{m+r}-\frac{2}{r-1} y_{m+1} \\
& +\frac{h^{3}\left(4 r^{4}-22 r^{3}+38 r^{2}-22 r+5\right)}{120 r} f_{m} \\
& -\frac{h^{3}\left(-14 r^{4}+55 r^{3}-55 r^{2}+5 r+5\right)}{60 r\left(r^{2}-3 r+2\right)} f_{m+r} \\
& -\frac{h^{3}\left(4 r^{4}-15 r^{3}+5 r^{2}+5 r-3\right)}{60(r-1)} f_{m+1} \\
& +\frac{h^{3}\left(4 r^{4}-8 r^{3}+2 r^{2}+2 r-1\right)}{120(r-2)} f_{m+2}, \\
& h y_{m+1}^{\prime}=-\frac{r-1}{r} y_{m}+\frac{1}{r(r-1)} y_{m+r}+\frac{r-2}{r-1} y_{m+1} \\
& -\frac{h^{3}\left(r^{4}-8 r^{3}+22 r^{2}-21 r+6\right)}{240 r} f_{m} \\
& +\frac{h^{3}\left(-r^{2}+2 r+3\right)}{120 r} f_{m+r} \\
& -\frac{h^{3}\left(-r^{3}+4 r^{2}+9 r-8\right)}{120} f_{m+1} \\
& -\frac{h^{3}\left(r^{3}-2 r+1\right)}{240} f_{m+2}, \\
& h^{2} y_{m+1}^{\prime \prime}=\frac{2}{r} y_{m}+\frac{2}{r(r-1)} y_{m+r}-\frac{2}{r-1} y_{m+1} \\
& -\frac{h^{3}\left(r^{4}-8 r^{3}+22 r^{2}-28 r+10\right)}{120 r} f_{m} \\
& -\frac{h^{3}\left(r^{4}-5 r^{3}+5 r^{2}+5 r-10\right)}{60 r\left(r^{2}-3 r+2\right)} f_{m+r} \\
& -\frac{h^{3}\left(-r^{4}+5 r^{3}+5 r^{2}-35 r+22\right)}{60(r-1)} f_{m+1} \\
& +\frac{h^{3}\left(-r^{4}+2 r^{3}+2 r^{2}-8 r+4\right)}{120(r-2)} f_{m+2} \text {. }
\end{aligned}
$$

\section{Characteristics of the Method}

This section is presented the basic properties of the main method and analyzed it 
to establish their validity.

\subsection{Order of the Method}

We can rewrite the hybrid block method in the form

$$
A Y_{m}=h B Y_{m}^{\prime}+h^{2} C Y_{m}^{\prime \prime}+h^{3} V F_{m}
$$

where $A, B, C, V$ are matrices of coefficients of dimensions $9 \times 4$,

$$
\begin{aligned}
& Y_{m}=\left(y_{m}, y_{m+r}, y_{m+1}, y_{m+2}\right)^{\mathrm{T}}, \\
& Y_{m}^{\prime}=\left(y_{m}^{\prime}, y_{m+r}^{\prime}, y_{m+1}^{\prime}, y_{m+2}^{\prime}\right)^{\mathrm{T}}, \\
& Y_{m}^{\prime \prime}=\left(y_{m}^{\prime \prime}, y_{m+r}^{\prime \prime}, y_{m+1}^{\prime \prime}, y_{m+2}^{\prime \prime}\right)^{\mathrm{T}}, \\
& F_{m}=\left(f_{m}, f_{m+r}, f_{m+1}, f_{m+2}\right)^{\mathrm{T}} .
\end{aligned}
$$

If $z(x)$ is a sufficiently differentiable function, the linear difference operator $\mathcal{L}$ associated with the implicit two-step block hybrid method is considered in Equation (8), Equation (9), Equation (10), Equation (13), that is given

$$
\begin{aligned}
\mathcal{L}\left[z\left(x_{m}\right) ; h\right]= & \sum_{j} \kappa_{j} z\left(x_{m}+j h\right)-h \tau_{j} z^{\prime}\left(x_{m}+j h\right) \\
& -h^{2} \gamma_{j} z^{\prime \prime}\left(x_{m}+j h\right)-h^{3} \xi_{j} z^{\prime \prime \prime}\left(x_{m}+j h\right)
\end{aligned}
$$

$j=0, r, 1,2$ where the $\kappa_{j}, \tau_{j}, \gamma_{j}$ and $\xi_{j}$ are respectively the vector columns of the matrices $A, B, C$ and $V$.

Expanding $z\left(x_{m}+j h\right), z^{\prime}\left(x_{m}+j h\right), z^{\prime \prime}\left(x_{m}+j h\right)$ and $z^{\prime \prime \prime}\left(x_{m}+j h\right)$ in Taylor series about $x_{m}$ we get

$$
\mathcal{L}\left[z\left(x_{m}\right) ; h\right]=C_{0} y\left(x_{m}\right)+C_{1} h y^{\prime}\left(x_{m}\right)+C_{2} h^{2} y^{\prime \prime}\left(x_{m}\right)+\cdots+C_{q} h^{q} y^{(q)}\left(x_{m}\right)+\cdots
$$

with $C_{0}=C_{1}=C_{2}=\cdots=C_{6}=0$ and

$$
\begin{aligned}
C_{7}= & \left(9.437739543552612 \times 10^{-6},-9.617040306545119 \times 10^{-20},\right. \\
& -3.070200924962517 \times 10^{-4},-4.913128762599697 \times 10^{-4}, \\
& 2.144715335032775 \times 10^{-3}, 8.823816935161847 \times 10^{-5}, \\
& -4.504524331158401 \times 10^{-4}, 3.184040914146728 \times 10^{-3}, \\
& \left.-1.141813120360736 \times 10^{-2}\right)^{\mathrm{T}}
\end{aligned}
$$

Note that the proposed method has order $p=4$ at least [9].

\subsection{Zero Stability}

We can write the method as a vector As $h \rightarrow 0$ in Equation (14), we can write the method as a vector form.

$A_{0} Y_{m}-A_{1} Y_{m-1}=0$ where

$$
\begin{gathered}
Y_{m}=\left(y_{m+2}, y_{m+1}, y_{m+r}\right)^{\mathrm{T}}, Y_{m-1}=\left(y_{m}, y_{m-1}, y_{m+r-1}\right)^{\mathrm{T}} \\
A_{0}=\left[\begin{array}{ccc}
1 & 0.6017954608298602 & -1.471021404061736 \\
0 & 1 & 0 \\
0 & 0 & 1
\end{array}\right]
\end{gathered}
$$




$$
A_{1}=\left[\begin{array}{ccc}
0.1307740567681242 & 0 & 0 \\
1 & 0 & 0 \\
1 & 0 & 0
\end{array}\right]
$$

The first characteristic polynomial

$\rho(z)=\operatorname{det}\left[A_{0} z-A_{1}\right]=(z-1) z^{2}=0$, the roots of polynomials are $z_{1}=z_{2}=0 ; z_{3}=1$. Hence the block method is zero -stable [9].

\subsection{Consistency}

The block method has order $p=4$, in case $p \geq 1$, this is a sufficient condition to be consistent with the associated block method [10].

\subsection{Convergence}

We can establish the convergence of the two-step with three points hybrid block method if and only if it is consistent and zero stable [11].

\subsection{Region of Absolute Stability}

As we mentioned earlier, zero-stability is a concept of the numerical method behavior for $h \rightarrow 0$. To decide whether a numerical method will produce good results with a given value of $h>0$, we need a concept of stability that is different from zero-stability. In most numerical methods intended to solve problems of third order, the stability properties are usually analyzed by considering the linear equation given by the Dalquist test [11].

$$
y^{\prime \prime \prime}=-\lambda^{3} y(x)
$$

This problem has bounded solutions for $\lambda \geq 0$ that tend to zero for $x \rightarrow \infty$. We will define the region where the numerical method reproduces the manner of the exact solutions. Let us explain the procedure for obtaining such a region. Our method has nine equations in which there are four different terms of first derivatives: $y_{m}^{\prime}, y_{m+r}^{\prime}, y_{m+1}^{\prime}, y_{m+2}^{\prime}$, and second derivative: $y_{m}^{\prime \prime}, y_{m+r}^{\prime \prime}, y_{m+1}^{\prime \prime}, y_{m+2}^{\prime \prime}$ and one intermediate values $y_{m+r}$. Let us depict the procedure to gain such a region [12], We eliminated these terms from the equations system by using mathematica, and get a recurrence equation in the terms $y_{m}, y_{m+1}, y_{m+2}$. This recurrence equation reads

$$
P(z) y_{m+2}+Q(z) y_{m+1}+S(z) y_{m}=0,
$$

where $z=\lambda h$, and

$$
\begin{aligned}
P(z)= & -1.433653893592262 \times 10^{109}+5.520254762660736 \times 10^{107} z^{3} \\
& +3.674797669760869 \times 10^{106} z^{6} \\
Q(z)= & 5.734615574369057 \times 10^{109}-8.898486741828162 \times 10^{108} z^{3} \\
& +3.679066781172749 \times 10^{107} z^{6} \\
S(z)= & -4.300961680776793 \times 10^{109}+2.867307787184524 \times 10^{109} z \\
& -1.211231358386333 \times 10^{108} z^{3}-6.261663213347228 \times 10^{107} z^{4} \\
+ & 4.300976789540145 \times 10^{106} z^{6}
\end{aligned}
$$


Through its characteristic equation, we study the extent boundedness of their solutions to define the region of stability. The root causes of the characteristic equation

$$
\begin{aligned}
& \left(-1.433653893592262 \times 10^{109}+5.520254762660736 \times 10^{107} z^{3}\right. \\
& \left.+3.674797669760869 \times 10^{106} z^{6}\right) x^{2}+\left(5.734615574369057 \times 10^{109}\right. \\
& \left.-8.898486741828162 \times 10^{108} z^{3}+3.679066781172749 \times 10^{107} z^{6}\right) x \\
& +\left(-4.300961680776793 \times 10^{109}+2.867307787184524 \times 10^{109} z\right. \\
& -1.211231358386333 \times 10^{108} z^{3}-6.261663213347228 \times 10^{107} z^{4} \\
& \left.+4.300976789540145 \times 10^{106} z^{6}\right)=0
\end{aligned}
$$

are

$$
\begin{aligned}
& x_{1}=-\frac{2}{4509981306728599 z^{6}+67748616455391176 z^{3}-1759485240133103872} \\
& \times\left(\sqrt{\left(121468655749357324985383025377280 z^{12}+86645450698059554742358457188352 z^{10}\right.}\right. \\
& -6085569311397395324399777440858112 z^{9}-2666041011195242645961971549077504 z^{7} \\
& +125054287321797496835881016603181056 z^{6}-93404454773980569973608059370471424 z^{4} \\
& -936741366582786806117795003533099008 z^{3}+1547894155123122963374310449590829056 z \\
& +773947077561567679793163991204757504) \\
& \left.-546043788941838592 z^{3}+22576103367311352 z^{6}+3518970480266213376\right) \\
& x_{2}=\frac{2}{4509981306728599 z^{6}+67748616455391176 z^{3}-1759485240133103872} \\
& \times\left(\sqrt { ( } \left(121468655749357324985383025377280 z^{12}+86645450698059554742358457188352 z^{10}\right.\right. \\
& -6085569311397395324399777440858112 z^{9}-2666041011195242645961971549077504 z^{7} \\
& +125054287321797496835881016603181056 z^{6}-93404454773980569973608059370471424 z^{4} \\
& -936741366582786806117795003533099008 z^{3}+1547894155123122963374310449590829056 z \\
& +773947077561567679793163991204757504) \\
& \left.+546043788941838592 z^{3}-22576103367311352 z^{6}-3518970480266213376\right)
\end{aligned}
$$

The roots of the characteristic equation must be less than 1, for the method to be stable. The stability region for the method has shown in Figure 1.

\section{Numerical Examples}

Example 1: Consider the Jerk equation in the following form:

$$
y^{\prime \prime \prime}=-y^{\prime}+y y^{\prime} y^{\prime \prime}
$$

With initial conditions: $y(0)=0, y^{\prime}(0)=B, y^{\prime \prime}(0)=0$, and exact solution:

$$
y(x)=\frac{B}{\Omega} \sin (\Omega x)+\frac{B}{96 \Omega^{3}}\left(\left(-9 B^{2}-48+48 \Omega^{2}\right) \sin (\Omega x)-B^{2} \sin (3 \Omega x)\right)
$$

where $\Omega=\frac{1}{2} \sqrt{B^{2}+4}$.

Tables 1-3 show the absolute Errors at $h=0.125,0.0125$ and $B=0.2,0.3,0.4$ respectively. Example 1 was solved in [13], the comparison be- 
tween our method and result in [13] is proposed in Table 4 and Table 5. It is seen in Figures 2-4 the method gives a good approximation in the interval $x \in[0,50]$ and $B=0.2,0.3,0.4$ respectively.

Example 2: Consider the Jerk equation in the following form:

$$
y^{\prime \prime \prime}=-y^{\prime}-y^{\prime} y^{\prime \prime 2}
$$

With initial conditions: $y(0)=0, y^{\prime}(0)=B, y^{\prime \prime}(0)=0$, and exact solution:

$$
\begin{aligned}
y(x)= & \frac{B}{\Omega} \sin (\Omega x)+\frac{B}{96 \Omega^{3}}\left(\left(-9 B^{2} \Omega^{2}-48+48 \Omega^{2}\right) \sin (\Omega x)\right. \\
& \left.+\left(12 \Omega^{3} B^{2}+48 \Omega-48 \Omega^{3}\right) x \cos (\Omega x)-B^{2} \Omega^{2} \sin (3 \Omega x)\right)
\end{aligned}
$$

where $\Omega=2 \sqrt{\frac{1}{4-B^{2}}}$.

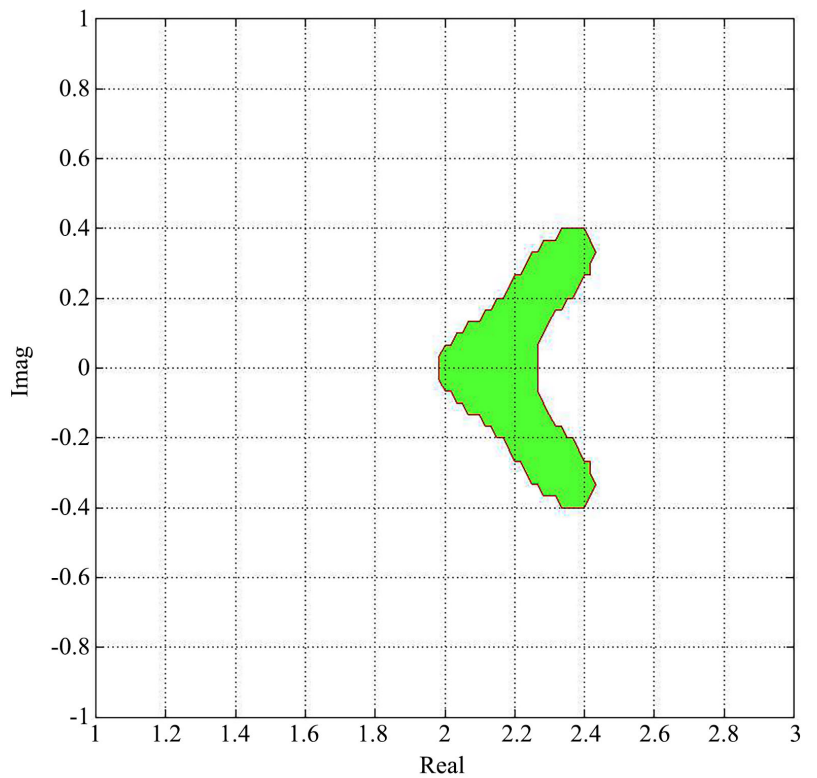

Figure 1. Region of absolute stability.

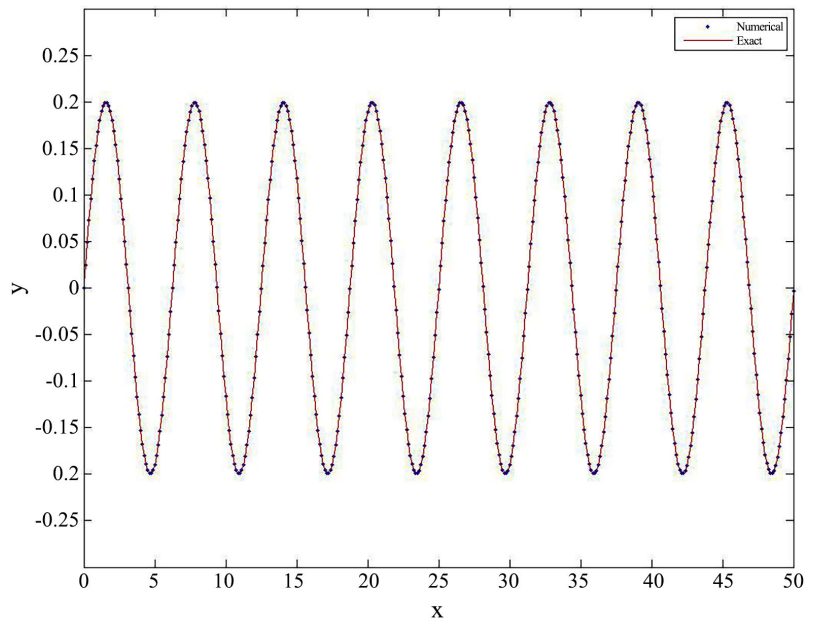

Figure 2. The solution of Example 1 at $h=0.125, \quad B=0.2$. 


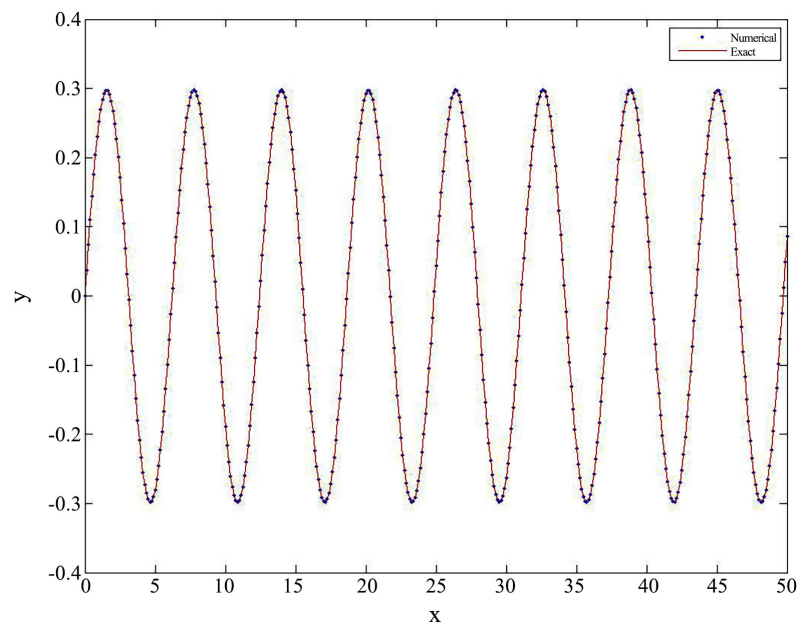

Figure 3. The solution of Example 1 at $h=0.125, \quad B=0.3$.

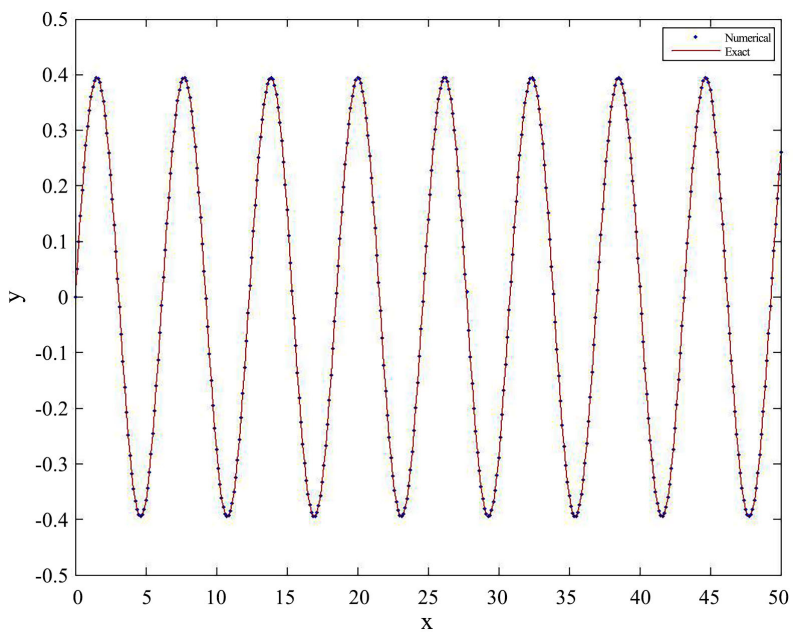

Figure 4. The solution of Example 1 at $h=0.125, \quad B=0.4$.

Table 1. Absolute error of numerical solutions at $B=0.2$.

\begin{tabular}{ccccccc}
\hline $\mathrm{X}$ & $\begin{array}{c}\text { Numerical Solution } \\
\text { in Our method at } \\
h=0.125\end{array}$ & $\begin{array}{c}\text { Exact Solution at } \\
h=0.125\end{array}$ & Error & $\begin{array}{c}\text { Numerical Solution } \\
\text { in Our method at } \\
h=0.0125\end{array}$ & $\begin{array}{c}\text { Exact Solution at } \\
h=0.0125\end{array}$ & Error \\
\hline 0 & 0 & 0 & 0 & 0 & 0 & 0.0249349 \\
0.125 & 0.024934 & 0.024934 & $4.6952 \times 10^{-13}$ & 0.024934 & $0.02495 \times 10^{-11}$ \\
0.25 & 0.049480 & 0.049480 & $1.09357 \times 10^{-9}$ & 0.049480 & 0.049480 & $1.39199 \times 10^{-9}$ \\
0.375 & 0.073253 & 0.073253 & $9.03272 \times 10^{-9}$ & 0.073253 & 0.073253 & $9.87559 \times 10^{-9}$ \\
0.5 & 0.095881 & 0.095881 & $3.59222 \times 10^{-8}$ & 0.095881 & 0.095881 & $3.81834 \times 10^{-8}$ \\
0.625 & 0.117008 & 0.117008 & $9.9039 \times 10^{-8}$ & 0.117008 & 0.117008 & $1.037 \times 10^{-7}$ \\
0.75 & 0.136300 & 0.1363 & $2.14523 \times 10^{-7}$ & 0.136300 & 0.1363 & $2.23488 \times 10^{-7}$ \\
0.875 & 0.153453 & 0.153452 & $3.91443 \times 10^{-7}$ & 0.153453 & 0.153452 & $4.0688 \times 10^{-7}$ \\
1 & 0.168191 & 0.168191 & $6.24133 \times 10^{-7}$ & 0.168191 & 0.168191 & $6.49333 \times 10^{-7}$ \\
1.125 & 0.180281 & 0.18028 & $8.91573 \times 10^{-7}$ & 0.180281 & 0.18028 & $9.29984 \times 10^{-7}$ \\
1.25 & 0.189526 & 0.189525 & $1.15844 \times 10^{-6}$ & 0.189526 & 0.189525 & $1.21443 \times 10^{-6}$ \\
1.375 & 0.195778 & 0.195777 & $1.38459 \times 10^{-6}$ & 0.195779 & 0.195777 & $1.46242 \times 10^{-6}$ \\
\hline
\end{tabular}


Continued

\begin{tabular}{cccccccc}
\hline 1.5 & 0.198937 & 0.198935 & $1.53404 \times 10^{-6}$ & 0.198937 & 0.198935 & $1.63819 \times 10^{-6}$ \\
1.625 & 0.198949 & 0.198948 & $1.58573 \times 10^{-6}$ & 0.198949 & 0.198948 & $1.72019 \times 10^{-6}$ \\
1.75 & 0.195816 & 0.195815 & $1.53899 \times 10^{-6}$ & 0.195817 & 0.195815 & $1.70703 \times 10^{-6}$ \\
1.875 & 0.189589 & 0.189587 & $1.41336 \times 10^{-6}$ & 0.189589 & 0.189587 & $1.61747 \times 10^{-6}$ \\
2 & 0.180367 & 0.180365 & $1.24351 \times 10^{-6}$ & 0.180367 & 0.180365 & $1.48465 \times 10^{-6}$ \\
\hline
\end{tabular}

Table 2. Absolute error of numerical solutions at $B=0.3$.

\begin{tabular}{ccccccc}
\hline$X$ & $\begin{array}{c}\text { Numerical Solution } \\
\text { in Our method at } \\
h=0.125\end{array}$ & $\begin{array}{c}\text { Exact Solution at } \\
h=0.125\end{array}$ & Error & $\begin{array}{c}\text { Numerical Solution } \\
\text { in Our method at } \\
h=0.0125\end{array}$ & $\begin{array}{c}\text { Exact Solution at } \\
h=0.0125\end{array}$ & Error \\
\hline 0 & 0 & 0 & 0 & 0 & 0 & 0 \\
0.125 & 0.037402 & 0.037402 & $6.28175 \times 10^{-10}$ & 0.037402 & 0.037402 & $3.43214 \times 10^{-10}$ \\
0.25 & 0.074220 & 0.074220 & $1.24193 \times 10^{-8}$ & 0.074220 & 0.074220 & $1.05686 \times 10^{-8}$ \\
0.375 & 0.109879 & 0.109878 & $7.99073 \times 10^{-8}$ & 0.109878 & 0.109878 & $7.55001 \times 10^{-8}$ \\
0.5 & 0.143814 & 0.143814 & $2.98246 \times 10^{-7}$ & 0.143814 & 0.143814 & $2.89694 \times 10^{-7}$ \\
0.625 & 0.17549 & 0.17549 & $7.99279 \times 10^{-7}$ & 0.17549 & 0.17549 & $7.86251 \times 10^{-7}$ \\
0.75 & 0.204399 & 0.204397 & $1.70916 \times 10^{-6}$ & 0.204399 & 0.204397 & $1.69292 \times 10^{-6}$ \\
0.875 & 0.230073 & 0.23007 & $3.09552 \times 10^{-6}$ & 0.230073 & 0.23007 & $3.07827 \times 10^{-6}$ \\
1 & 0.252093 & 0.252088 & $4.91735 \times 10^{-6}$ & 0.252093 & 0.252088 & $4.90448 \times 10^{-6}$ \\
1.125 & 0.270095 & 0.270088 & $7.01179 \times 10^{-6}$ & 0.270095 & 0.270088 & $7.00958 \times 10^{-6}$ \\
1.25 & 0.283778 & 0.283769 & $9.11212 \times 10^{-6}$ & 0.283778 & 0.283769 & $9.13002 \times 10^{-6}$ \\
1.375 & 0.292913 & 0.292902 & 0.000010 & 0.292913 & 0.292902 & 0.000010 \\
1.5 & 0.297344 & 0.297332 & 0.000012 & 0.297344 & 0.297332 & 0.000012 \\
1.625 & 0.296996 & 0.296984 & 0.000012 & 0.296996 & 0.296984 & 0.000012 \\
1.75 & 0.291876 & 0.291864 & 0.000012 & 0.291876 & 0.291864 & 0.000012 \\
1.875 & 0.28207 & 0.282059 & 0.000011 & 0.282071 & 0.282059 & 0.000011 \\
2 & 0.267745 & 0.267734 & 0.000010 & 0.267745 & 0.267734 & 0.000010 \\
\hline
\end{tabular}

Table 3. Absolute error of numerical solutions at $B=0.4$.

\begin{tabular}{ccccccc}
\hline$X$ & $\begin{array}{c}\text { Numerical Solution } \\
\text { in Our method at } \\
h=0.125\end{array}$ & $\begin{array}{c}\text { Exact Solution at } \\
h=0.125\end{array}$ & Error & $\begin{array}{c}\text { Numerical Solution } \\
\text { in Our method at } \\
h=0.0125\end{array}$ & $\begin{array}{c}\text { Exact Solution at } \\
h=0.0125\end{array}$ & Error \\
\hline 0 & 0 & 0 & 0 & 0 & 0 & 0 \\
0.125 & 0.049869 & 0.049869 & $2.46607 \times 10^{-9}$ & 0.049869 & 0.049869 & $1.4462 \times 10^{-9}$ \\
0.25 & 0.098960 & 0.098960 & $5.11558 \times 10^{-8}$ & 0.098961 & 0.098961 & $4.45237 \times 10^{-8}$ \\
0.375 & 0.146501 & 0.146501 & $3.34007 \times 10^{-7}$ & 0.146501 & 0.146501 & $3.17797 \times 10^{-7}$ \\
0.5 & 0.191739 & 0.191738 & $1.25262 \times 10^{-6}$ & 0.191739 & 0.191738 & $1.21922 \times 10^{-6}$ \\
0.625 & 0.233947 & 0.233943 & $3.36066 \times 10^{-6}$ & 0.233947 & 0.233943 & $3.30604 \times 10^{-6}$ \\
0.75 & 0.272436 & 0.272428 & $7.18675 \times 10^{-6}$ & 0.272436 & 0.272428 & $7.10926 \times 10^{-6}$ \\
0.875 & 0.306566 & 0.306553 & 0.000013 & 0.306566 & 0.306553 & 0.000012 \\
1 & 0.33576 & 0.335739 & 0.000021 & 0.33576 & 0.335739 & 0.000020 \\
1.125 & 0.359513 & 0.359483 & 0.000029 & 0.359513 & 0.359483 & 0.000029 \\
1.25 & 0.377408 & 0.37737 & 0.000038 & 0.377408 & 0.37737 & 0.000037 \\
1.375 & 0.389127 & 0.389082 & 0.000045 & 0.389127 & 0.389082 & 0.000045 \\
1.5 & 0.39446 & 0.394409 & 0.000050 & 0.39446 & 0.394409 & 0.000050 \\
\hline
\end{tabular}


Continued

\begin{tabular}{ccccccc}
\hline 1.625 & 0.393308 & 0.393256 & 0.000052 & 0.393308 & 0.393256 & 0.000052 \\
1.75 & 0.385695 & 0.385643 & 0.000051 & 0.385695 & 0.385643 & 0.000051 \\
1.875 & 0.371756 & 0.371707 & 0.000048 & 0.371756 & 0.371707 & 0.000048 \\
2 & 0.351742 & 0.351698 & 0.000044 & 0.351742 & 0.351698 & 0.000044 \\
\hline
\end{tabular}

Table 4. Comparison between the numerical solution in our method and the method in [13], $B=0.2, h=0.125$.

\begin{tabular}{ccc}
\hline$X$ & Numerical Solution in our method & Numerical Solution in [13] \\
\hline 0 & 0 & 0.024934 \\
0.125 & 0.024934 & 0.049480 \\
0.25 & 0.049480 & 0.073254 \\
0.375 & 0.073253 & 0.095885 \\
0.5 & 0.095881 & 0.117019 \\
0.625 & 0.117008 & 0.136327 \\
0.75 & 0.1363 & 0.153508 \\
0.875 & 0.153453 & 0.168294 \\
1 & 0.168191 & 0.180453 \\
1.125 & 0.180281 & 0.189796 \\
1.25 & 0.189526 & 0.196178 \\
1.375 & 0.195778 & 0.199498 \\
1.5 & 0.198937 & 0.199706 \\
1.625 & 0.198949 & 0.196797 \\
1.75 & 0.195816 & 0.190817 \\
1.875 & 0.189589 & 0.181859 \\
2 & 0.180367 & \\
\end{tabular}

Table 5. Comparsion between the numerical solution in our method and the method in [13], $B=0.4, h=0.125$.

\begin{tabular}{ccc}
\hline$X$ & Numerical Solution in our method & Numerical Solution in [13] \\
\hline 0 & 0 & 0.049869 \\
0.125 & 0.049869 & 0.098961 \\
0.25 & 0.098960 & 0.146509 \\
0.375 & 0.146501 & 0.191770 \\
0.5 & 0.191739 & 0.234038 \\
0.625 & 0.233947 & 0.272655 \\
0.75 & 0.272436 & 0.307017 \\
0.875 & 0.306566 & 0.336588 \\
1 & 0.33576 & 0.360907 \\
1.125 & 0.359513 & 0.379593 \\
1.25 & 0.377408 & 0.392357 \\
1.375 & 0.389127 & 0.398997 \\
1.5 & 0.39446 & 0.399412 \\
1.625 & 0.393308 & 0.393594 \\
1.75 & 0.385695 & 0.381634 \\
1.875 & 0.371756 & 0.363718 \\
\hline
\end{tabular}


Tables 6-8 show the absolute Errors at $h=0.125,0.0125$ and $B=0.2,0.3,0.4$ respectively. Example 2 was solved in [13], the comparison between our method and result in [13] is proposed in Table 9 and Table 10. It is seen in Figures 5-7 the method gives a good approximation in the interval $x \in[0,50]$ and $B=0.2,0.3,0.4$ respectively.

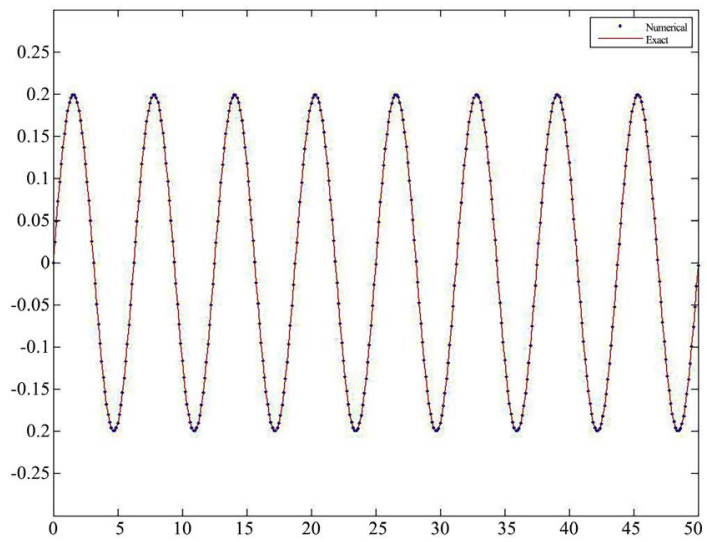

Figure 5. The solution of Example 2 at $h=0.125, \quad B=0.2$.

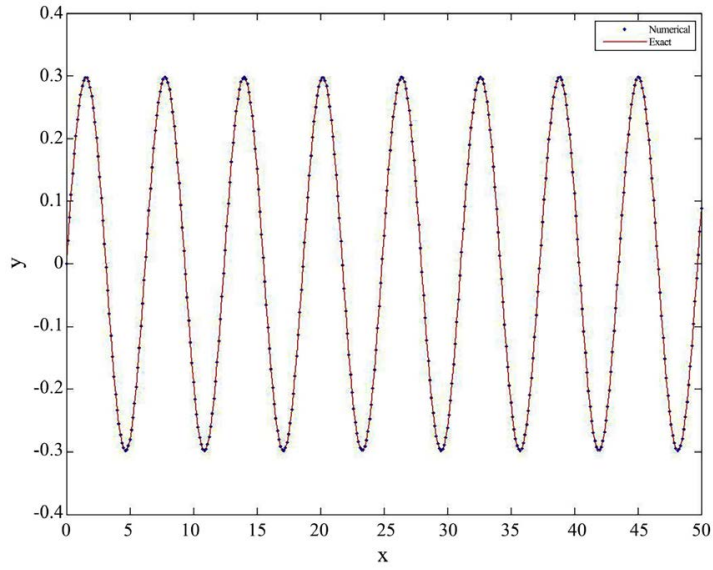

Figure 6. The solution of Example 2 at $h=0.125, \quad B=0.3$.

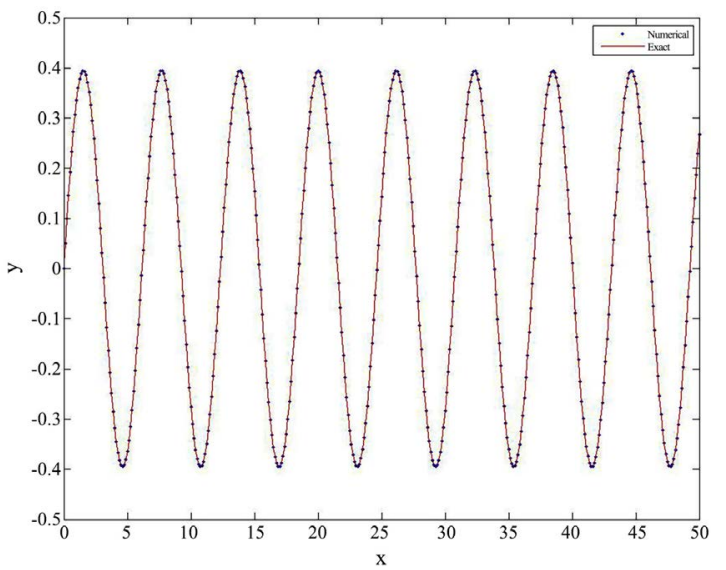

Figure 7. The solution of Example 2 at $h=0.125, B=0.4$. 
Table 6. Absolute error of numerical solutions at $B=0.2$.

\begin{tabular}{|c|c|c|c|c|c|c|}
\hline$X$ & $\begin{array}{c}\text { Numerical Solution in } \\
\text { Our method at } \\
h=0.125\end{array}$ & $\begin{array}{l}\text { Exact Solution at } \\
\quad h=0.125\end{array}$ & Error & $\begin{array}{c}\text { Numerical Solution } \\
\text { in Our method at } \\
h=0.0125\end{array}$ & $\begin{array}{l}\text { Exact Solution at } \\
\quad h=0.0125\end{array}$ & Error \\
\hline 0 & 0 & 0 & 0 & 0 & 0 & 0 \\
\hline 0.125 & 0.024934 & 0.024934 & $3.89579 \times 10^{-11}$ & 0.024934 & 0.024934 & $8.68102 \times 10^{-11}$ \\
\hline 0.25 & 0.049480 & 0.049480 & $2.37202 \times 10^{-9}$ & 0.049480 & 0.049480 & $2.68466 \times 10^{-9}$ \\
\hline 0.375 & 0.073253 & 0.073253 & $1.83415 \times 10^{-8}$ & 0.073253 & 0.073253 & $1.92163 \times 10^{-8}$ \\
\hline 0.5 & 0.095881 & 0.095881 & $7.26168 \times 10^{-8}$ & 0.095881 & 0.095881 & $7.49309 \times 10^{-8}$ \\
\hline 0.625 & 0.117008 & 0.117008 & $2.01662 \times 10^{-7}$ & 0.117008 & 0.117008 & $2.06392 \times 10^{-7}$ \\
\hline 0.75 & 0.1363 & 0.1363 & $4.44143 \times 10^{-7}$ & 0.1363 & 0.1363 & $4.53178 \times 10^{-7}$ \\
\hline 0.875 & 0.153452 & 0.153452 & $8.29786 \times 10^{-7}$ & 0.153452 & 0.153452 & $8.45282 \times 10^{-7}$ \\
\hline 1 & 0.168191 & 0.16819 & $1.3665 \times 10^{-6}$ & 0.168191 & 0.16819 & $1.39175 \times 10^{-6}$ \\
\hline 1.125 & 0.18028 & 0.180278 & $2.03637 \times 10^{-6}$ & 0.18028 & 0.180278 & $2.07483 \times 10^{-6}$ \\
\hline 1.25 & 0.189525 & 0.189522 & $2.79641 \times 10^{-6}$ & 0.189525 & 0.189522 & $2.85251 \times 10^{-6}$ \\
\hline 1.375 & 0.195777 & 0.195773 & $3.59097 \times 10^{-6}$ & 0.195777 & 0.195773 & $3.66902 \times 10^{-6}$ \\
\hline 1.5 & 0.198934 & 0.198929 & $4.36561 \times 10^{-6}$ & 0.198934 & 0.198929 & $4.47017 \times 10^{-6}$ \\
\hline 1.625 & 0.198945 & 0.19894 & $5.08301 \times 10^{-6}$ & 0.198945 & 0.19894 & $5.21817 \times 10^{-6}$ \\
\hline 1.75 & 0.195811 & 0.195805 & $5.73169 \times 10^{-6}$ & 0.195811 & 0.195805 & $5.90079 \times 10^{-6}$ \\
\hline 1.875 & 0.189581 & 0.189575 & $6.32606 \times 10^{-6}$ & 0.189581 & 0.189575 & $6.53163 \times 10^{-6}$ \\
\hline 2 & 0.180357 & 0.18035 & $6.89847 \times 10^{-6}$ & 0.180357 & 0.18035 & $7.14149 \times 10^{-6}$ \\
\hline
\end{tabular}

Table 7. Absolute error of numerical solutions at $B=0.3$.

\begin{tabular}{|c|c|c|c|c|c|c|}
\hline$X$ & $\begin{array}{c}\text { Numerical Solution } \\
\text { in Our method at } \\
h=0.125\end{array}$ & $\begin{array}{l}\text { Exact Solution at } \\
\qquad h=0.125\end{array}$ & Error & $\begin{array}{l}\text { Numerical Solution } \\
\text { in Our method at } \\
h=0.0125\end{array}$ & $\begin{array}{c}\text { Exact Solution at } \\
\quad h=0.0125\end{array}$ & Error \\
\hline 0 & 0 & 0 & 0 & 0 & 0 & 0 \\
\hline 0.125 & 0.037402 & 0.037402 & $9.40558 \times 10^{-10}$ & 0.037402 & 0.037402 & $6.72264 \times 10^{-10}$ \\
\hline 0.25 & 0.074220 & 0.074220 & $2.25371 \times 10^{-8}$ & 0.074220 & 0.074220 & $2.07935 \times 10^{-8}$ \\
\hline 0.375 & 0.109879 & 0.109878 & $1.53599 \times 10^{-7}$ & 0.109878 & 0.109878 & $1.49427 \times 10^{-7}$ \\
\hline 0.5 & 0.143814 & 0.143814 & $5.88902 \times 10^{-7}$ & 0.143814 & 0.143814 & $5.80723 \times 10^{-7}$ \\
\hline 0.625 & 0.17549 & 0.175489 & $1.61265 \times 10^{-6}$ & 0.17549 & 0.175489 & $1.60006 \times 10^{-6}$ \\
\hline 0.75 & 0.204399 & 0.204395 & $3.52997 \times 10^{-6}$ & 0.204399 & 0.204395 & $3.51404 \times 10^{-6}$ \\
\hline 0.875 & 0.230072 & 0.230066 & $6.57212 \times 10^{-6}$ & 0.230072 & 0.230066 & $6.55487 \times 10^{-6}$ \\
\hline 1 & 0.252091 & 0.25208 & 0.000010 & 0.252091 & 0.25208 & 0.000010 \\
\hline 1.125 & 0.27009 & 0.270074 & 0.000016 & 0.27009 & 0.270074 & 0.000016 \\
\hline 1.25 & 0.283769 & 0.283747 & 0.000022 & 0.283769 & 0.283747 & 0.000022 \\
\hline 1.375 & 0.292898 & 0.29287 & 0.000028 & 0.292898 & 0.29287 & 0.000028 \\
\hline 1.5 & 0.297321 & 0.297287 & 0.000034 & 0.297321 & 0.297287 & 0.000034 \\
\hline 1.625 & 0.296964 & 0.296924 & 0.000040 & 0.296964 & 0.296924 & 0.000040 \\
\hline 1.75 & 0.291832 & 0.291786 & 0.000045 & 0.291832 & 0.291786 & 0.000045 \\
\hline 1.875 & 0.282012 & 0.281962 & 0.000050 & 0.282013 & 0.281962 & 0.000050 \\
\hline 2 & 0.267672 & 0.267617 & 0.000054 & 0.267672 & 0.267617 & 0.000055 \\
\hline
\end{tabular}


Table 8. Absolute error of numerical solutions at $B=0.4$.

\begin{tabular}{|c|c|c|c|c|c|c|}
\hline$X$ & $\begin{array}{l}\text { Numerical Solution } \\
\text { in Our method at } \\
h=0.125\end{array}$ & $\begin{array}{l}\text { Exact Solution at } \\
\quad h=0.125\end{array}$ & Error & $\begin{array}{l}\text { Numerical Solution } \\
\text { in Our method at } \\
h=0.0125\end{array}$ & $\begin{array}{c}\text { Exact Solution at } \\
\quad h=0.0125\end{array}$ & Error \\
\hline 0 & 0 & 0 & 0 & 0 & 0 & 0 \\
\hline 0.125 & 0.049869 & 0.049869 & $3.86139 \times 10^{-9}$ & 0.049869 & 0.049869 & $2.91296 \times 10^{-9}$ \\
\hline 0.25 & 0.098960 & 0.098960 & $9.62982 \times 10^{-8}$ & 0.098960 & 0.09896 & $9.01239 \times 10^{-8}$ \\
\hline 0.375 & 0.146501 & 0.146501 & $6.62938 \times 10^{-7}$ & 0.146501 & 0.146501 & $6.47732 \times 10^{-7}$ \\
\hline 0.5 & 0.191739 & 0.191736 & $2.55096 \times 10^{-6}$ & 0.191739 & 0.191736 & $2.51913 \times 10^{-6}$ \\
\hline 0.625 & 0.233946 & 0.233939 & $6.99674 \times 10^{-6}$ & 0.233946 & 0.233939 & $6.9439 \times 10^{-6}$ \\
\hline 0.75 & 0.272434 & 0.272419 & 0.000015 & 0.272434 & 0.272419 & 0.000015 \\
\hline 0.875 & 0.306562 & 0.306533 & 0.000028 & 0.306561 & 0.306533 & 0.000028 \\
\hline 1 & 0.335749 & 0.335702 & 0.000046 & 0.335749 & 0.335702 & 0.000046 \\
\hline 1.125 & 0.359492 & 0.359422 & 0.000069 & 0.359492 & 0.359422 & 0.000069 \\
\hline 1.25 & 0.37737 & 0.377275 & 0.000095 & 0.37737 & 0.377275 & 0.000095 \\
\hline 1.375 & 0.389065 & 0.388942 & 0.000122 & 0.389065 & 0.388942 & 0.000122 \\
\hline 1.5 & 0.394363 & 0.394214 & 0.000149 & 0.394363 & 0.394214 & 0.000149 \\
\hline 1.625 & 0.393169 & 0.392994 & 0.000174 & 0.393169 & 0.392994 & 0.000174 \\
\hline 1.75 & 0.385503 & 0.385307 & 0.000196 & 0.385504 & 0.385307 & 0.000196 \\
\hline 1.875 & 0.371507 & 0.371289 & 0.000217 & 0.371507 & 0.371289 & 0.000217 \\
\hline 2 & 0.351432 & 0.351193 & 0.000238 & 0.351432 & 0.351193 & 0.000238 \\
\hline
\end{tabular}

Table 9. Comparsion between the numerical solution in our method and the method in [13], $B=0.2, h=0.125$.

\begin{tabular}{ccc}
\hline$X$ & Numerical Solution in our method & Numerical Solution in [13] \\
\hline 0 & 0 & 0.024934 \\
0.125 & 0.024934 & 0.049480 \\
0.25 & 0.049480 & 0.073254 \\
0.375 & 0.073253 & 0.095885 \\
0.5 & 0.095881 & 0.117019 \\
0.625 & 0.117008 & 0.136327 \\
0.75 & 0.1363 & 0.153508 \\
0.875 & 0.153452 & 0.168294 \\
1 & 0.168191 & 0.180453 \\
1.125 & 0.18028 & 0.189796 \\
1.25 & 0.189525 & 0.196178 \\
1.375 & 0.195777 & 0.199498 \\
1.5 & 0.198934 & 0.199706 \\
1.625 & 0.198945 & 0.196797 \\
1.75 & 0.195811 & 0.190817 \\
1.875 & 0.189581 & 0.181859 \\
\hline
\end{tabular}


Table 10. Comparison between the numerical solution in our method and the method in [13], $B=0.4$.

\begin{tabular}{|c|c|c|}
\hline$X$ & Numerical Solution in our method & Numerical Solution in [13] \\
\hline 0 & 0 & 0 \\
\hline 0.125 & 0.049869 & 0.049869 \\
\hline 0.25 & 0.098960 & 0.098961 \\
\hline 0.375 & 0.146501 & 0.146509 \\
\hline 0.5 & 0.191739 & 0.191770 \\
\hline 0.625 & 0.233946 & 0.234038 \\
\hline 0.75 & 0.272434 & 0.272655 \\
\hline 0.875 & 0.306562 & 0.307017 \\
\hline 1 & 0.335749 & 0.336588 \\
\hline 1.125 & 0.359492 & 0.360907 \\
\hline 1.25 & 0.37737 & 0.379593 \\
\hline 1.375 & 0.389065 & 0.392357 \\
\hline 1.5 & 0.394363 & 0.398997 \\
\hline 1.625 & 0.393169 & 0.399412 \\
\hline 1.75 & 0.385503 & 0.393594 \\
\hline 1.875 & 0.371507 & 0.381634 \\
\hline 2 & 0.351432 & 0.363718 \\
\hline
\end{tabular}

\section{Conclusion}

A two-step with one hybrid point was proposed and proceeded as a self-starting method for solving nonlinear Jerk equations. We considered one hybrid point and specified for approximation after optimizing local truncation errors related to the main formula. Therefore, our method's good convergent and stability properties make it attractive for the numerical solution of nonlinear problems. The method presented is zero stable, consistent and convergence of four-algebraic order. The numerical results and figures show their efficiency and precision compared to other methods in the literature.

\section{Conflicts of Interest}

The authors declare no conflicts of interest regarding the publication of this paper.

\section{References}

[1] Schot, S.H. (1978) Jerk: The Time Rate of Change of Acceleration. American Journal of Physics, 46, 1090-1094. https://doi.org/10.1119/1.11504

[2] Anu, N. and Marinca, V. (2016) Approximate Analytical Solutions to Jerk Equations. Dynamical Systems. Theoretical and Experimental Analysis, Łódź, 7-10 December 2015, 169-176. https://doi.org/10.1007/978-3-319-42408-8 14

[3] Gottlieb, H. (2004) Harmonic Balance Approach to Periodic Solutions of Non-Linear 
Jerk Equations. Journal of Sound and Vibration, 271, 671-683. https://doi.org/10.1016/S0022-460X(03)00299-2

[4] Momoniat, E. and Mahomed, F. (2010) Symmetry Reduction and Numerical Solution of a Third-Order ODE from Thin Film Flow. Mathematical and Computational Applications, 15, 709-719. https://doi.org/10.3390/mca15040709

[5] Bhrawy, A. and Abd-Elhameed, W. (2011) New Algorithm for the Numerical Solutions of Nonlinear Third-Order Differential Equations Using Jacobi-Gauss Collocation Method. Mathematical Problems in Engineering, 2011, Article ID: 837218. https://doi.org/10.1155/2011/837218

[6] Raftari, B. (2013) He's Variational Iteration Method for Nonlinear Jerk Equations: Simple but Effective. Shock and Vibration, 20, 351-356. https://doi.org/10.1155/2013/493048

[7] Rahman, M.S. and Hasan, A. (2018) Modified Harmonic Balance Method for the Solution of Nonlinear Jerk Equations. Results in Physics, 8, 893-897. https://doi.org/10.1016/j.rinp.2018.01.030

[8] Larson, R. and Falvo, D. (2004) Elementary Linear Algebra. 6th Edition.

[9] Lambert, J.D. and Lambert, J. (1973) Computational Methods in Ordinary Differential Equations. Wiley, London, 23.

[10] Jator, S.N. (2007) A Sixth Order Linear Multistep Method for the Direct Solution of $y^{\prime \prime}=f\left(x, y, y^{\prime}\right)$. International Journal of Pure and Applied Mathematics, 40, 457-472.

[11] Dahlquist, G. (1956) Convergence and Stability in the Numerical Integration of Ordinary Differential Equations. Mathematica Scandinavica, 4, 33-53. https://doi.org/10.7146/math.scand.a-10454

[12] Ramos, H., Kalogiratou, Z., Monovasilis, T. and Simos, T. (2016) An Optimized Two-Step Hybrid Block Method for Solving General Second Order Initial-Value Problems. Numerical Algorithms, 72, 1089-1102.

https://doi.org/10.1007/s11075-015-0081-8

[13] Mirzabeigy, A. and Yildirim, A. (2014) Approximate Periodic Solution for Nonlinear Jerk Equation as a Third-Order Nonlinear Equation via Modified Differential Transform Method. Engineering Computations, 31, 622-633. https://doi.org/10.1108/EC-02-2012-0024 